\title{
A CHARACTERIZATION OF HYBRIDIZED MIXED METHODS FOR SECOND ORDER ELLIPTIC PROBLEMS
}

\author{
BERNARDO COCKBURN AND JAYADEEP GOPALAKRISHNAN
}

This paper is dedicated to Jim Douglas, Jr., on the occasion of his $75^{\text {th }}$ birthday.

\begin{abstract}
In this paper, we give a new characterization of the approximate solution given by hybridized mixed methods for second-order, self-adjoint elliptic problems. We apply this characterization to obtain an explicit formula for the entries of the matrix equation for the Lagrange multiplier unknowns resulting from hybridization. We also obtain necessary and sufficient conditions under which the multipliers of the Raviart-Thomas and the Brezzi-DouglasMarini methods of similar order are identical.
\end{abstract}

\section{INTRODUCTION}

In this paper, we give a new characterization of hybridized mixed methods. This characterization allows us to obtain an explicit formula for the entries of the matrix equation for the so-called Lagrange multipliers. It also allows comparison of hybridized versions of different mixed methods. For example, we give conditions under which the multipliers of the Raviart-Thomas method and those of the BrezziDouglas-Marini method of comparable order coincide.

We consider the hybridized version [1] of the standard Raviart-Thomas mixed method [11] for the elliptic boundary value problem

$$
\begin{aligned}
-\nabla \cdot(a \nabla u)+d u & =f & & \text { in } \Omega \subset \mathbb{R}^{2}, \\
u & =g & & \text { on } \partial \Omega,
\end{aligned}
$$

where $a(\boldsymbol{x})$ is a symmetric positive definite matrix-valued function, $d(\boldsymbol{x})$ is a nonnegative function and $\Omega$ is a polygonal domain in $\mathbb{R}^{2}$. We assume that $a(\boldsymbol{x})$ and $d(\boldsymbol{x})$ are bounded. We consider this simple setting for transparent presentation of the main ideas. As will be clear later, our techniques can be applied to other hybridized methods and more general second order elliptic problems.

Before describing the results, recall that mixed finite element methods seek approximations $\left(\boldsymbol{q}_{h}, u_{h}\right)$ to $(a \nabla u, u)$ in appropriate finite element spaces. They give rise to a matrix equation of the form

$$
\left(\begin{array}{cc}
\mathbb{A} & -\mathbb{B}^{t} \\
\mathbb{B} & \mathbb{D}
\end{array}\right)\left(\begin{array}{l}
\mathbb{Q} \\
\mathbb{U}
\end{array}\right)=\left(\begin{array}{l}
\mathbb{G} \\
\mathbb{F}
\end{array}\right)
$$

Received by the editor November 13, 2002.

1991 Mathematics Subject Classification. Primary 54C40, 14E20; Secondary 46E25, 20C20.

Key words and phrases. mixed finite elements, hybrid methods, elliptic problems.

The first author was partially supported by the National Science Foundation (Grant DMS0107609) and by the University of Minnesota Supercomputer Institute. 
where $\mathbb{Q}$ and $\mathbb{U}$ are the vectors of coefficients of $\boldsymbol{q}_{h}$ and $u_{h}$ with respect to their corresponding finite element basis, respectively. Since the system is not positive definite, solving for $\mathbb{Q}$ and $\mathbb{U}$ is not always easy. Although one can arrive at a positive definite system by elimination of $\mathbb{Q}$ from the equations, this requires inversion of $\mathbb{A}$ and maintaining $\mathbb{A}^{-1}$ which is typically a full matrix. Fortunately, by 'hybridizing' the mixed method, this difficulty can be overcome. Let us now briefly recall the hybridization procedure.

First, the so-called Lagrange multiplier $\lambda_{h}$ is introduced which gives rise to a matrix equation of the form

$$
\left(\begin{array}{ccc}
A & -B^{t} & -C^{t} \\
B & D & 0 \\
C & 0 & 0
\end{array}\right)\left(\begin{array}{l}
Q \\
U \\
\Lambda
\end{array}\right)=\left(\begin{array}{c}
\mathcal{G} \\
\mathcal{F} \\
0
\end{array}\right),
$$

where $\Lambda$ is the vector of degrees of freedom associated to the multiplier $\lambda_{h}$. We will precisely state the underlying finite element spaces later. As is now well-known, the new vectors of degrees of freedom $Q$ and $U$ actually define the same approximation $\left(\boldsymbol{q}_{h}, u_{h}\right)$ as the original mixed method. Moreover, both $Q$ and $U$ can now be easily eliminated to obtain an equation for the multiplier only, namely,

$$
\mathbb{E} \Lambda=\mathbb{H},
$$

where $\mathbb{E}$ and $\mathbb{H}$ are given by

$$
\begin{aligned}
& \mathbb{E}=C A^{-1}\left(A-B^{t}\left(B A^{-1} B^{t}+D\right)^{-1} B\right) A^{-1} C^{t}, \\
& \mathbb{H}=\mathbb{H}_{g}+\mathbb{H}_{f}, \\
& \mathbb{H}_{g}=-C A^{-1}\left(A-B^{t}\left(B A^{-1} B^{t}+D\right)^{-1} B\right) A^{-1} \mathcal{G}, \\
& \mathbb{H}_{f}=-C A^{-1} B^{t}\left(B A^{-1} B^{t}+D\right)^{-1} \mathcal{F} .
\end{aligned}
$$

That the inverses taken above exist follow from the properties of the underlying finite element spaces. Considering this matrix equation instead of the previous one has several advantages: (i) the matrix $\mathbb{E}$ is symmetric and positive definite, so it can be numerically inverted by using methods like the Conjugate Gradient method, (ii) the number of degrees of freedom of the multiplier is remarkably smaller than the number of degrees of freedom of the original mixed method, (iii) once $\Lambda$ has been obtained, both $Q$ and $U$ can be efficiently computed element by element, and (iv) the multiplier $\lambda_{h}$ can actually be used to improve the approximation to $u$ by means of a local post-processing, as shown in [1]. This shows that the use of hybridized mixed methods is indeed very advantageous; however, the complicated relation between the matrices $\mathbb{E}$ and $\mathbb{H}$, and the matrices $A, B, C, \mathcal{F}$ and $\mathcal{G}$, can easily dissuade one to base an implementation on $\mathbb{E}$ and $\mathbb{H}$.

In this paper, we show that the entries of the matrices $\mathbb{E}$ and $\mathbb{H}$ can be expressed as a weighted $L^{2}$-innerproduct of some discontinuous auxiliary functions, the weights being nothing but the matrix $a^{-1}$ and the function $d$. These auxiliary functions are easily constructed in terms of the geometry of the mesh, the matrix $a$, the function $d$, and the spaces of the hybridized mixed finite element method. Their definition induces a natural decomposition of the approximate solution $\left(\boldsymbol{q}_{h}, u_{h}\right)$ of the form

$$
\left(\boldsymbol{q}_{h}, u_{h}\right)=\left(\boldsymbol{q}_{h}, u_{h}\right)_{\lambda_{h}}+\left(\boldsymbol{q}_{h}, u_{h}\right)_{g}+\left(\boldsymbol{q}_{h}, u_{h}\right)_{f},
$$

where $\left(\boldsymbol{q}_{h}, u_{h}\right)_{\lambda_{h}}$ is a lifting of the Lagrange multiplier $\lambda_{h}$, and $\left(\boldsymbol{q}_{h}, u_{h}\right)_{g}$ and $\left(\boldsymbol{q}_{h}, u_{h}\right)_{f}$ can be computed locally only in terms of the data. Introduction of other 
discrete lifting operators have proved useful in another context earlier, namely the analysis of discontinuous Galerkin methods for elliptic problems $[2,7,8]$.

We then present two applications of this result. As a first application, we present a technique to assemble the matrix of the Lagrange multiplier equation using simple local element matrices. Next, we compare the matrices $\mathbb{E}$ and $\mathbb{H}$ of the RaviartThomas method with those of the Brezzi-Douglas-Marini method of similar order and give necessary and sufficient conditions for the multipliers to be the exactly the same. This happens, for example, when $d=0$ and $f=0$, a case that occurs in many situations of practical interest.

The paper is organized as follows. In Section 2, we introduce the hybridized version of the mixed method of Raviart and Thomas and then state, discuss and prove the characterization result, Theorem 2.1. In Section 3, we show how to assemble the matrices for the multipliers and in Section 4, we compare the matrices of the Raviart-Thomas method with those of the Brezzi-Douglas-Marini method of similar order. Finally, in Section 5, we end with some concluding remarks.

\section{THE MAIN RESULT}

We begin this Section by introducing the classical mixed method of Raviart and Thomas [11]; then, following [1], we hybridize the method. Finally, we state, discuss, and prove the main result, Theorem 2.1.

2.1. The hybridized mixed method. Given a triangulation of $\Omega, \mathcal{T}_{h}$, made of triangles, the mixed method seeks an approximation $\left(\boldsymbol{q}_{h}, u_{h}\right)$ to the solution $(\boldsymbol{q}, u)$ of the model problem

$$
\begin{aligned}
c \boldsymbol{q} & =-\nabla u & & \text { in } \Omega, \\
\nabla \cdot \boldsymbol{q}+d u & =f & & \text { in } \Omega, \\
u & =g & & \text { on } \partial \Omega,
\end{aligned}
$$

where $c=a^{-1}$. The approximation $\left(\boldsymbol{q}_{h}, u_{h}\right)$ is sought in the finite element space $\mathbb{V}_{h} \times \mathbb{W}_{h}$ given by

$$
\begin{array}{ll}
\mathbb{V}_{h}=\{\boldsymbol{v} \in H(\operatorname{div}, \Omega): & \left.\left.\boldsymbol{v}\right|_{K} \in P^{k}(K) \times P^{k}(K)+\boldsymbol{x} P^{k}(K), \quad \text { for all } K \in \mathcal{T}_{h}\right\}, \\
\mathbb{W}_{h}=\left\{w \in L^{2}(\Omega):\right. & \left.\left.w\right|_{K} \in P^{k}(K), \quad \text { for all } K \in \mathcal{T}_{h}\right\},
\end{array}
$$

where $P^{k}(K)$ denotes the space of polynomials on $K$ of degree at most $k$, and is defined by requiring that, for all $(\boldsymbol{v}, w) \in V_{h} \times W_{h}$,

$$
\begin{aligned}
& \int_{\Omega} c \boldsymbol{q}_{h} \cdot \boldsymbol{v} d x-\int_{\Omega} u_{h} \nabla \cdot \boldsymbol{v} d x=-\int_{\partial \Omega} g \boldsymbol{v} \cdot \boldsymbol{n} d s, \\
& \int_{\Omega} w \nabla \cdot \boldsymbol{q}_{h} d x+\int_{\Omega} d u_{h} w d x=\int_{\Omega} f w d x .
\end{aligned}
$$

It is easy to see that the above weak formulation gives rise to a system of equations of the form

$$
\left(\begin{array}{cc}
\mathbb{A} & -\mathbb{B}^{t} \\
\mathbb{B} & \mathbb{D}
\end{array}\right)\left(\begin{array}{l}
\mathbb{Q} \\
\mathbb{U}
\end{array}\right)=\left(\begin{array}{l}
\mathbb{G} \\
\mathbb{F}
\end{array}\right)
$$

We can try to solve this equation by first eliminating $\mathbb{Q}$ from the equations and then solving the resulting equation for $\mathbb{U}$, namely,

$$
\left(\mathbb{B} \mathbb{A}^{-1} \mathbb{B}^{t}+\mathbb{D}\right) \mathbb{U}=\mathbb{F}+\mathbb{B} \mathbb{A}^{-1} \mathbb{G} .
$$


Unfortunately, the matrix $\mathbb{A}$ is not easy to invert since the elements of $V_{h}$, being functions in $H(\operatorname{div}, \Omega)$, have their normal component continuous across element interfaces. If $\boldsymbol{q}_{h}$ were totally discontinuous, $\mathbb{A}$ would be block-diagonal and hence easily invertible. The idea of the hybridized mixed methods is to relax this continuity constraint to render $\mathbb{A}$ block-diagonal.

Indeed, it was Fraejis deVeubeque [9], back in 1965, who realized that this can be achieved by introducing additional unknowns $\lambda_{h}$, associated to element interfaces, called Lagrange multipliers. Let $\mathcal{E}_{i, h}$ denote the set of edges of the mesh $\mathcal{T}_{h}$ that are in the interior of the domain $\Omega$. The multipliers are then nothing but approximations to the trace of $u$ on each $e \in \mathcal{E}_{i, h}$. As we show next, their introduction allows elimination of both $\boldsymbol{q}_{h}$ and $u_{h}$ and reduction of the system to a single matrix equation for the multipliers.

In our particular case, the hybridized mixed method seeks an approximation $\left(\boldsymbol{q}_{h}, u_{h}, \lambda_{h}\right)$ to $\left(\boldsymbol{q}, u,\left.u\right|_{\mathcal{E}_{i, h}}\right)$ in the finite element space $V_{h} \times W_{h} \times M_{h}$ given by

$V_{h}=\left\{\boldsymbol{v} \in L^{2}(\Omega) \times L^{2}(\Omega):\left.\quad \boldsymbol{v}\right|_{K} \in P^{k}(K) \times P^{k}(K)+\boldsymbol{x} P^{k}(K), \quad\right.$ for all $\left.K \in \mathcal{T}_{h}\right\}$,

$W_{h}=\left\{w \in L^{2}(\Omega):\left.\quad w\right|_{K} \in P^{k}(K), \quad\right.$ for all $\left.K \in \mathcal{T}_{h}\right\}$,

$M_{h}=\left\{\mu \in L^{2}\left(\mathcal{E}_{i, h}\right):\left.\quad \mu\right|_{e} \in P^{k}(e), \quad\right.$ for all $\left.e \in \mathcal{E}_{i, h}\right\}$.

It is defined by requiring that, for all $(\boldsymbol{v}, u, \mu) \in V_{h} \times W_{h} \times M_{h}$,

$$
\begin{aligned}
& \int_{\Omega} c \boldsymbol{q}_{h} \cdot \boldsymbol{v} d x-\sum_{K \in \mathcal{T}_{h}} \int_{K} u_{h} \nabla \cdot \boldsymbol{v} d x+\sum_{e \in \mathcal{E}_{i, h}} \int_{e} \lambda_{h} \llbracket \boldsymbol{v} \rrbracket d s=-\int_{\partial \Omega} g \llbracket \boldsymbol{v} \rrbracket d s, \\
& \sum_{K \in \mathcal{T}_{h}} \int_{K} w \nabla \cdot \boldsymbol{q}_{h} d x+\int_{\Omega} d u_{h} w d x=\int_{\Omega} f w d x \\
& \sum_{e \in \mathcal{E}_{i, h}} \int_{e} \mu \llbracket \boldsymbol{q}_{h} \rrbracket d s=0,
\end{aligned}
$$

where, $\llbracket \boldsymbol{v} \rrbracket=\boldsymbol{v} \cdot \boldsymbol{n}$ on $\partial \Omega$, and $\llbracket \boldsymbol{v} \rrbracket=\boldsymbol{v}_{e}^{+} \cdot \boldsymbol{n}_{e}^{+}+\boldsymbol{v}_{e}^{-} \cdot \boldsymbol{n}_{e}^{-}$, on $e \in \mathcal{E}_{h}$. Here, $\boldsymbol{n}$ denotes the outward unit normal to $\Omega, \boldsymbol{n}_{e}^{+}=-\boldsymbol{n}_{e}^{-}$is an arbitrary unit vector normal to the $e \in \mathcal{E}_{i, h}$ and $\boldsymbol{v}_{e}^{ \pm}(\boldsymbol{x})=\lim _{\epsilon \downarrow 0} \boldsymbol{v}\left(\boldsymbol{x}-\epsilon \boldsymbol{n}_{e}^{ \pm}\right)$.

2.2. Two lifting operators. Next, we introduce two mappings in terms of which the characterization result will be expressed. They are defined using the equations (2.6) and (2.7).

The first mapping lifts functions on edges of the triangulation $\mathcal{T}_{h}$ to functions on $\Omega$. Let $\mathcal{E}_{h}$ be the set of all edges of the triangulation $\mathcal{T}_{h}$. Notwithstanding a slight abuse of notation, we shall denote the set of all square integrable functions on the union of all edges of $\mathcal{E}_{h}$ by $L^{2}\left(\mathcal{E}_{h}\right)$. The first lifting associates to each $\mathrm{m} \in L^{2}\left(\mathcal{E}_{h}\right)$, the pair of functions $\left(\boldsymbol{q}_{h}, u_{h}\right)_{\mathrm{m}} \equiv\left(\boldsymbol{q}_{h, \mathrm{~m}}, u_{h, \mathrm{~m}}\right) \in V_{h} \times W_{h}$ defined by requiring that

$$
\begin{aligned}
& \int_{\Omega} c \boldsymbol{q}_{h, \mathrm{~m}} \cdot \boldsymbol{v} d x-\sum_{K \in \mathcal{T}_{h}} \int_{K} u_{h, \mathrm{~m}} \nabla \cdot \boldsymbol{v} d x=-\sum_{e \in \mathcal{E}_{h}} \int_{e} \mathrm{~m} \llbracket \boldsymbol{v} \rrbracket d s, \\
& \sum_{K \in \mathcal{T}_{h}} \int_{K} w \nabla \cdot \boldsymbol{q}_{h, \mathrm{~m}} d x+\int_{\Omega} d u_{h, \mathrm{~m}} w d x=0
\end{aligned}
$$

hold for all $(\boldsymbol{v}, w) \in V_{h} \times W_{h}$. 
The second lifting, associates to the function $f \in L^{2}(\Omega)$ the element $\left(\boldsymbol{q}_{h}, u_{h}\right)_{f} \equiv$ $\left(\boldsymbol{q}_{h, f}, u_{h, f}\right) \in V_{h} \times W_{h}$ and is defined by requiring that

$$
\begin{aligned}
& \int_{\Omega} c \boldsymbol{q}_{h, f} \cdot \boldsymbol{v} d x-\sum_{K \in \mathcal{T}_{h}} \int_{K} u_{h, f} \nabla \cdot \boldsymbol{v} d x=0, \\
& \sum_{K \in \mathcal{T}_{h}} \int_{K} w \nabla \cdot \boldsymbol{q}_{h, f} d x+\int_{\Omega} d u_{h, f} w d x=\int_{\Omega} f w d x,
\end{aligned}
$$

hold for all $(\boldsymbol{v}, w) \in V_{h} \times W_{h}$.

Note that these liftings can be computed in an element-by-element fashion. Indeed, the liftings are uniquely defined on each element because of the surjectivity of the map $(\nabla \cdot): V_{h} \mapsto W_{h}$ restricted to an element. Moreover, on each element $K \in \mathcal{T}_{h}$, the lifting $\left(\boldsymbol{q}_{h}, u_{h}\right)_{\mathrm{m}}$ can be thought of as a result of a one element discretization of the following boundary value problem

$$
\begin{aligned}
c \boldsymbol{q}_{\mathrm{m}} & =-\nabla u_{\mathrm{m}} & & \text { in } K, \\
\nabla \cdot \boldsymbol{q}_{\mathrm{m}}+d u_{\mathrm{m}} & =0 & & \text { in } K, \\
u_{\mathrm{m}} & =\mathrm{m} & & \text { on } \partial K,
\end{aligned}
$$

and that the lifting $\left(\boldsymbol{q}_{h}, u_{h}\right)_{f}$ is an approximation to the solution of

$$
\begin{aligned}
c \boldsymbol{q}_{f} & =-\nabla u_{f} & & \text { in } K, \\
\nabla \cdot \boldsymbol{q}_{f}+d u_{f} & =f & & \text { in } K, \\
u_{f} & =0 & & \text { on } \partial K .
\end{aligned}
$$

2.3. Characterization of the approximate solution. Before stating the result, let us introduce the following convention: The extension by zero of the function $\eta \in L^{2}\left(\mathcal{F}_{h}\right)$, where $\mathcal{F}_{h}$ is a subset of $\mathcal{E}_{h}$, to $\mathcal{E}_{h}$ is also denoted by $\eta$. In this way, if $\mathrm{m}=\lambda_{h}$ on $\mathcal{E}_{i, h}$ and $\mathrm{m}=g$ on $\partial \Omega$, we simply write $\mathrm{m}=\lambda_{h}+g$; as a consequence we also write

$$
\left(\boldsymbol{q}_{h}, u_{h}\right)_{\mathrm{m}}=\left(\boldsymbol{q}_{h}, u_{h}\right)_{\lambda_{h}}+\left(\boldsymbol{q}_{h}, u_{h}\right)_{g} .
$$

We now have all that is needed to state the main result.

Theorem 2.1 (Characterization of $\left.\left(\boldsymbol{q}_{h}, u_{h}, \lambda_{h}\right)\right)$. Let $\left(\boldsymbol{q}_{h}, u_{h}, \lambda_{h}\right)$ be the solution of the hybridized Raviart-Thomas method (2.6), (2.7) and (2.8). Then

$$
\left(\boldsymbol{q}_{h}, u_{h}\right)=\left(\boldsymbol{q}_{h}, u_{h}\right)_{\lambda_{h}}+\left(\boldsymbol{q}_{h}, u_{h}\right)_{g}+\left(\boldsymbol{q}_{h}, u_{h}\right)_{f} .
$$

The Lagrange multiplier $\lambda_{h} \in M_{h}$ is the unique solution of

$$
a_{h}\left(\lambda_{h}, \mu\right)=b_{h}(\mu) \quad \text { for all } \mu \in M_{h},
$$

where

and

$$
a_{h}\left(\lambda_{h}, \mu\right)=\int_{\Omega} c \boldsymbol{q}_{h, \lambda_{h}} \cdot \boldsymbol{q}_{h, \mu} d x+\int_{\Omega} d u_{h, \lambda_{h}} u_{h, \mu} d x
$$

$$
b_{h}(\mu)=\int_{\partial \Omega} g \llbracket \boldsymbol{q}_{h, \mu} \rrbracket d s+\int_{\Omega} f u_{h, \mu} d x .
$$

Remark 2.1. Although the normal components of the functions $\boldsymbol{q}_{h, g}, \boldsymbol{q}_{h, f}$ (which can be computed locally only in terms of the data) and $\boldsymbol{q}_{h, \lambda_{h}}$ are not necessarily continuous across inter-element boundaries, the normal components of their sum, namely $\boldsymbol{q}_{h}$, are. Marini [10] pointed out this fact for the lowest order RaviartThomas method when $d=0$ and $a$ is a piecewise-constant scalar function. 
Remark 2.2. Just like for classical finite element methods, the variational formulation (2.13) gives rise to a matrix equation for the degrees of freedom of the multiplier $\Lambda$ of the form

$$
\mathbb{E} \Lambda=\mathbb{H} \text {. }
$$

Thus, the entries of the matrices $\mathbb{E}$ and $\mathbb{H}$ are obtained as a weighted $L^{2}$-innerproduct of discontinuous functions, as claimed in the introduction.

We end this section with a proof of Theorem 2.1.

2.4. Proof of Theorem 2.1. We prove this result in three steps. In the first, we observe some identities that result from the equations of the method. In the next step, we show that the continuity condition on the jumps of the fluxes results in a variational equation for the Lagrange multiplier unknowns. In the last step, we collect these two results and conclude.

\subsubsection{Step 1.}

Lemma 2.1 (Elementary identities). We have, for any $\mathrm{m}, \mu \in L^{2}\left(\mathcal{E}_{h}\right)$ and $f \in$ $L^{2}(\Omega)$,

$$
\begin{aligned}
& \text { (i) }-\sum_{e \in \mathcal{E}_{h}} \int_{e} \mu \llbracket \boldsymbol{q}_{h, \mathrm{~m}} \rrbracket d s=\int_{\Omega} c \boldsymbol{q}_{h, \mathrm{~m}} \cdot \boldsymbol{q}_{h, \mu} d x+\int_{\Omega} d u_{h, \mathrm{~m}} u_{h, \mu} d x, \\
& (i i)-\sum_{e \in \mathcal{E}_{h}} \int_{e} \mathrm{~m} \llbracket \boldsymbol{q}_{h, f} \rrbracket d s=-\int_{\Omega} f u_{h, \mathrm{~m}} d x, \\
& \text { (iii) } \int_{\Omega} c \boldsymbol{q}_{h, f} \cdot \boldsymbol{q}_{h, \mathrm{~m}} d x+\int_{\Omega} d u_{h, f} u_{h, \mathrm{~m}} d x=0 .
\end{aligned}
$$

Proof. Let us first prove the identity (i). First, take $\boldsymbol{v}=\boldsymbol{q}_{h, \mu}$ in equation (2.9). Then, replace $\mathrm{m}$ by $\mu$ in equation (2.10) and take $w=u_{h, \mathrm{~m}}$. The identity (i) follows by simply adding these two equations.

Next, let us prove (ii). Take $\boldsymbol{v}=\boldsymbol{q}_{h, f}$ in equation (2.9). Now, if we take $w=u_{h, \mathrm{~m}}$ in (2.12) and add the two equations, we get

$$
-\sum_{e \in \mathcal{E}_{h}} \int_{e} \mathrm{~m} \llbracket \boldsymbol{q}_{h, f} \rrbracket d s=-\int_{\Omega} f u_{h, \mathrm{~m}} d x+\Theta,
$$

where

$$
\Theta=\int_{\Omega} c \boldsymbol{q}_{h, f} \cdot \boldsymbol{q}_{h, \mathrm{~m}} d x+\int_{\Omega} d u_{h, f} u_{h, \mathrm{~m}} d x .
$$

Thus, (ii) follows from (iii).

Finally, to prove (iii), simply take $w=u_{h, f}$ in equation (2.10), $\boldsymbol{v}=\boldsymbol{q}_{h, \mathrm{~m}}$ in equation (2.11) and add the equations. This completes the proof.

2.4.2. Step 2. In the following lemma, we explore equivalent characterizations of the continuity requirement on fluxes imposed by the method.

Lemma 2.2 (Jump condition). Let $\left(\boldsymbol{q}_{h}, u_{h}, \lambda_{h}\right)$ be the solution of the hybridized Raviart-Thomas method (2.6), (2.7) and (2.8), and let $\mathrm{m}$ be an arbitrary member of $M_{h}$. Then the following statements are equivalent:

(1) $\sum_{e \in \mathcal{E}_{i, h}} \int_{e} \mu \llbracket \boldsymbol{q}_{h, \mathrm{~m}}+\boldsymbol{q}_{h, g}+\boldsymbol{q}_{h, f} \rrbracket d s=0 \quad$ for all $\mu \in M_{h}$.

(2) $\left(\boldsymbol{q}_{h}, u_{h}\right)=\left(\boldsymbol{q}_{h}, u_{h}\right)_{\mathrm{m}}+\left(\boldsymbol{q}_{h}, u_{h}\right)_{g}+\left(\boldsymbol{q}_{h}, u_{h}\right)_{f}$,

(3) $\mathrm{m}=\lambda_{h}$. 
(4) $a_{h}(\mathrm{~m}, \mu)=b_{h}(\mu) \quad$ for all $\mu \in M_{h}$.

Proof. (1) $\Longrightarrow(2)$ : Set

$$
\left(\widetilde{\boldsymbol{q}}_{h}, \widetilde{u}_{h}\right)=\left(\boldsymbol{q}_{h}, u_{h}\right)_{\mathrm{m}}+\left(\boldsymbol{q}_{h}, u_{h}\right)_{g}+\left(\boldsymbol{q}_{h}, u_{h}\right)_{f} .
$$

Then combining the equations defining the liftings we get that

$$
\begin{aligned}
& \int_{\Omega} c \widetilde{\boldsymbol{q}}_{h} \cdot \boldsymbol{v} d x-\sum_{K \in \mathcal{T}_{h}} \int_{K} \widetilde{u}_{h} \nabla \cdot \boldsymbol{v} d x+\sum_{e \in \mathcal{E}_{i, h}} \int_{e} \mathrm{~m} \llbracket \boldsymbol{v} \rrbracket d s=-\int_{\partial \Omega} g \llbracket \boldsymbol{v} \rrbracket d s, \\
& \sum_{K \in \mathcal{T}_{h}} \int_{K} w \nabla \cdot \widetilde{\boldsymbol{q}}_{h} d x+\int_{\Omega} d \widetilde{u}_{h} w d x=\int_{\Omega} f w d x,
\end{aligned}
$$

for all $(\boldsymbol{v}, w) \in V_{k} \times W_{h}$. Therefore, whenever (1) holds, $\widetilde{\boldsymbol{q}}_{h}$ and $\widetilde{u}_{h}$ satisfy all the equations of the hybridized Raviart-Thomas method. By uniqueness of solutions of the method, $\widetilde{\boldsymbol{q}}_{h}=\boldsymbol{q}_{h}$ and $\widetilde{u}_{h}=u_{h}$, so (2) follows.

$(2) \Longrightarrow(3)$ : By linear superposition, $\boldsymbol{q}_{h}=\boldsymbol{q}_{h, \lambda_{h}}+\boldsymbol{q}_{h, g}+\boldsymbol{q}_{h, f}$. Moreover $\int_{e} \mu \llbracket \boldsymbol{q}_{h} \rrbracket d s=0$ for all $e \in \mathcal{E}_{i, h}$. From the implication (1) $\Longrightarrow(2)$, it follows that

$$
\left(\boldsymbol{q}_{h}, u_{h}\right)=\left(\boldsymbol{q}_{h}, u_{h}\right)_{\lambda_{h}}+\left(\boldsymbol{q}_{h}, u_{h}\right)_{g}+\left(\boldsymbol{q}_{h}, u_{h}\right)_{f} .
$$

Consequently, (2) implies that

$$
\left(\boldsymbol{q}_{h}, u_{h}\right)_{\mathrm{m}-\lambda_{h}}=0,
$$

from which it follows that $\mathrm{m}-\lambda_{h}=0$.

$(3) \Longrightarrow(4)$ : Now we observe that the following identity holds for any $\mathrm{m} \in M_{h}$ :

$$
\begin{gathered}
-\int_{\Omega} c \boldsymbol{q}_{h, \mathrm{~m}} \cdot \boldsymbol{q}_{h, \mu} d x-\int_{\Omega} d u_{h, \mathrm{~m}} u_{h, \mu} d x+\int_{\partial \Omega} g \llbracket \boldsymbol{q}_{h, \mu} \rrbracket d s+\int_{\Omega} f u_{h, \mu} d x \\
=\sum_{e \in \mathcal{E}_{h}} \int_{e} \mu \llbracket \boldsymbol{q}_{h, \mathrm{~m}}+\boldsymbol{q}_{h, g}+\boldsymbol{q}_{h, f} \rrbracket d s, \quad \text { for all } \mu \in M_{h} .
\end{gathered}
$$

It follows from the identities (i) and (iii) of Lemma 2.1. Moreover, whenever $\mathrm{m}=\lambda_{h}$, the last equation of the hybridized mixed method asserts that the right hand side of (2.14) is zero. From the definition of the forms $a_{h}$ and $b_{h}$ in Theorem (2.1), we see that (4) follows.

$(4) \Longrightarrow(1)$ : We apply (2.14) again. Whenever (4) holds, the left hand side of (2.14) is zero. Therefore, (1) follows.

2.4.3. Step 3. To conclude the proof the Theorem 2.1 observe that first assertion of the theorem follows from the equivalence of Items (1) and (2) of Lemma 2.2. The second assertion of the theorem follows again from Lemma 2.2, this time from the equivalence of (3) and (4). This completes the proof of Theorem 2.1.

Remark 2.3. The characterization theorem we just proved states that the solution $\left(\boldsymbol{q}_{h}, u_{h}\right)$ is the sum of the lifting of $\mathbf{m}=\lambda_{h}+g,\left(\boldsymbol{q}_{h}, u_{h}\right)_{\mathbf{m}}$, and the lifting of $f,\left(\boldsymbol{q}_{h}, u_{h}\right)_{f}$. By the identity (iii) of Lemma 2.1, we see that these liftings are orthogonal with respect to the bilinear form on $\left(V_{h} \times W_{h}\right)^{2}$ defined by

$$
\left\langle\left\langle\left(\boldsymbol{q}_{1}, u_{1}\right),\left(\boldsymbol{q}_{2}, u_{2}\right)\right\rangle\right\rangle=\int_{\Omega} c \boldsymbol{q}_{1} \cdot \boldsymbol{q}_{2} d x+\int_{\Omega} d u_{1} u_{2} d x
$$




\section{The MATRIX ENTRIES}

3.1. The local matrices. To compute the matrices $\mathbb{E}$ and $\mathbb{H}$, we can proceed in the traditional finite element way. Let $e$ denote an interior edge of the mesh $\mathcal{T}_{h}$, and let $\left\{L_{e, \ell}\right\}_{\ell=0}^{k}$ denote a basis for the set of polynomials of degree at most $k$ on $e$. For example, we can choose, properly scaled Legendre polynomials. Then, we set

$$
\left(\boldsymbol{q}_{e, \ell}, u_{e, \ell}\right)=\left(\boldsymbol{q}_{h}, u_{h}\right)_{L_{e, \ell}} .
$$

Now, for each element $K$, we compute the so-called local matrices whose entries are

$$
\begin{aligned}
\mathbb{E}_{K ; e, \ell ; e^{\prime}, \ell^{\prime}} & =\int_{K} c \boldsymbol{q}_{e, \ell} \cdot \boldsymbol{q}_{e^{\prime}, \ell^{\prime}} d x+\int_{K} d u_{e, \ell} u_{e^{\prime}, \ell^{\prime}} d x, \\
\mathbb{H}_{g_{K} ; e, \ell} & =\int_{\partial K \cap \partial \Omega} g \boldsymbol{q}_{e, \ell} \cdot \boldsymbol{n} d x, \\
\mathbb{H}_{f_{K ; e, \ell}} & =\int_{K} u_{e, \ell} f d x .
\end{aligned}
$$

Then the global matrices can be easily assembled by noting that

$$
\begin{aligned}
\Lambda_{e, \ell}^{t} \mathbb{E} \Lambda_{e^{\prime}, \ell^{\prime}} & =\sum_{K \in \mathcal{T}_{h}} \mathbb{E}_{K ; e, \ell ; e^{\prime}, \ell^{\prime}} \\
\Lambda_{e, \ell}^{t} \mathbb{H}_{g} & =\sum_{K \in \mathcal{T}_{h}} \mathbb{H}_{g_{K ; e, \ell}} \\
\Lambda_{e, \ell}^{t} \mathbb{H}_{f} & =\sum_{K \in \mathcal{T}_{h}} \mathbb{H}_{f K ; e, \ell}
\end{aligned}
$$

Since the lifting $\left(\boldsymbol{q}_{e, \ell}, u_{e, \ell}\right)$ is supported only on the triangles sharing the edge $e$, to compute the local matrices, we only have to provide the numbers $\mathbb{E}_{K ; e, \ell ; e^{\prime}, \ell^{\prime}}$, $\mathbb{H}_{g_{K ; e, \ell}}$ and $\mathbb{H}_{f K ; e, \ell}$ for any two edges $e$ and $e^{\prime}$ of $K$ and any two integers $\ell$ and $\ell^{\prime}$ between 0 and $k$; all the remaining entries are equal to zero. This also implies that the matrix $\mathbb{E}$ is a matrix of $(k+1) \times(k+1)$ blocks which has at most four off-diagonal blocks in each block column.

3.2. An example. The above computations can easily be carried out for the hybridized version of the Raviart-Thomas method of lowest order. In this example, as the subscript $\ell$ in $\boldsymbol{q}_{e, \ell}$ and $u_{e, \ell}$ is superfluous, we drop it.

We begin by computing the lifting $\mathrm{m} \mapsto\left(\boldsymbol{q}_{h}, u_{h}\right)_{\mathrm{m}}$. Let $\mathrm{m}$ take the constant value $\lambda_{i}$ on the edge $e_{i}$ of the triangle $K, i=1,2,3$. Then, on $K$,

$$
\left(\boldsymbol{q}_{h}, u_{h}\right)_{\mathrm{m}}=\sum_{i=1}^{3}\left(\boldsymbol{q}_{e_{i}}, u_{e_{i}}\right) \lambda_{i}
$$

where, for $\boldsymbol{x} \in K$ and $i=1,2,3$,

$$
\boldsymbol{q}_{e_{i}}(\boldsymbol{x})=-\frac{\left|e_{i}\right|}{|K|}(\bar{c})^{-1} \boldsymbol{n}_{i}-\frac{1}{2}\left(\frac{\bar{d} \rho_{i}}{1+\bar{d} \mathrm{~h}^{2}}\right)(\boldsymbol{x}-\boldsymbol{B}), \quad u_{e_{i}}(\boldsymbol{x})=\frac{\rho_{i}}{1+\bar{d} \mathrm{~h}^{2}},
$$

and

$$
\begin{aligned}
& \bar{c}=\frac{1}{|K|} \int_{K} c d x, \quad \bar{d}=\frac{1}{|K|} \int_{K} d d x, \quad \rho_{i}=\frac{\left|e_{i}\right|\left(\boldsymbol{m}_{i}-\boldsymbol{B}\right) \cdot \boldsymbol{n}_{i}}{2|K|}, \\
& \mathrm{h}^{2}=\frac{1}{4|K|} \int_{K} c(\boldsymbol{x}-\boldsymbol{B}) \cdot(\boldsymbol{x}-\boldsymbol{B}) d x, \quad \boldsymbol{B}=(\bar{c})^{-1} \frac{\int_{K} c \boldsymbol{x} d x}{|K|} .
\end{aligned}
$$




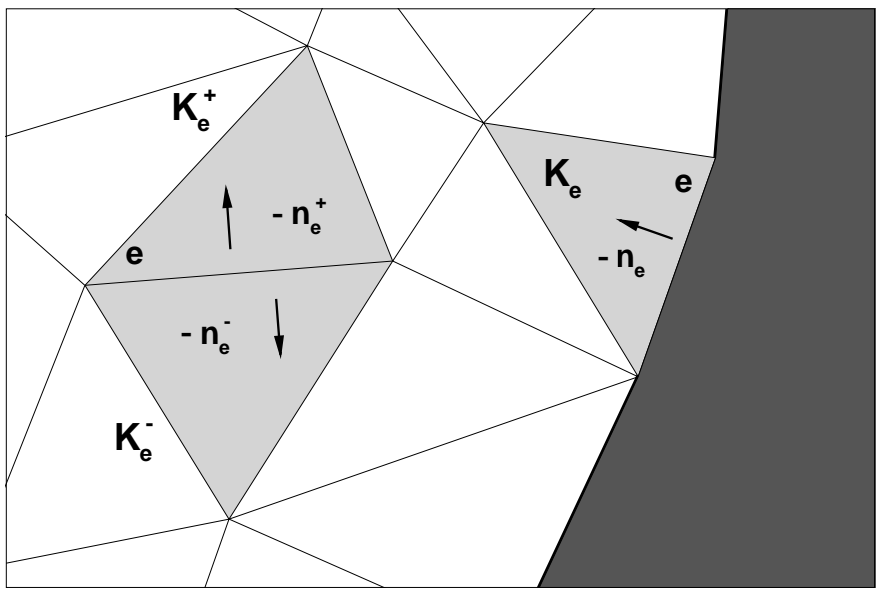

Figure 1. A representation of the lifting $\boldsymbol{q}_{h, \mathrm{~m}}$ when $\mathrm{m}=\chi_{e}$ for interior and boundary edges $e$. In this case, we have taken $d=0$ and $c=I d$.

Here $\boldsymbol{m}_{i}$ denotes the midpoint of the edge $e_{i}$ and $\boldsymbol{n}_{i}$ its outward unit normal. Note that if $c$ is the identity, $\boldsymbol{B}$ is the barycenter of the triangle; if, moreover, $K$ is an equilateral triangle of diameter $h$ then $\rho_{i}=1 / 3$ and $h^{2}=h^{2} / 48$. The case of $c$ equaling the $2 \times 2$ identity and vanishing $d$ is illustrated in Fig. 1 .

Now, it is easy to compute the entries of the local matrices:

$$
\begin{aligned}
\mathbb{E}_{K ; e_{i} ; e_{j}} & =\frac{\left|e_{i}\right|\left|e_{j}\right|}{|K|} \boldsymbol{n}_{j} \cdot(\bar{c})^{-1} \boldsymbol{n}_{j}+\frac{\bar{d}|K|}{1+\bar{d} \mathrm{~h}^{2}} \rho_{i} \rho_{j}, \\
\mathbb{H}_{g_{K ; e_{i}}} & =-\sum_{e_{j} \subset \partial K \cap \partial \Omega}\left(\frac{\left|e_{i}\right|}{|K|}(\bar{c})^{-1} \boldsymbol{n}_{i} \cdot \boldsymbol{n}_{j}+\frac{|K|}{\left|e_{j}\right|}\left(\frac{\bar{d}}{1+\bar{d} \mathrm{~h}^{2}}\right) \rho_{i} \rho_{j}\right) \int_{e_{j}} g(s) d s, \\
\mathbb{H}_{f_{K ; e_{i}}} & =\frac{\rho_{i}}{1+\bar{d} \mathrm{~h}^{2}} \int_{K} f(x) d x .
\end{aligned}
$$

3.3. The reference element. A convenient implementation results if the local matrices for the multiplier can be computed by using quadratures on a reference element alone.

To achieve it, we need to define liftings on the reference element, and map spaces on the reference element $\widehat{K}$ to corresponding ones on any triangle $K$. Let $\widehat{K}$ be mapped one-to-one onto $K$ by the affine mapping

$$
\boldsymbol{x}=D_{K} \widehat{\boldsymbol{x}}+b_{K},
$$

and let us set

$$
\widehat{u}(\widehat{\boldsymbol{x}}) \equiv u(\boldsymbol{x}), \quad \widehat{\boldsymbol{q}}(\widehat{\boldsymbol{x}}) \equiv\left|\operatorname{det} D_{K}\right| D_{K}^{-1} \boldsymbol{q}(\boldsymbol{x}),
$$

for scalar-valued functions $u$, vector-valued functions $\boldsymbol{q}$, respectively. Finally, set

$$
\begin{aligned}
& \widehat{V}=P^{k}(\widehat{K}) \times P^{k}(\widehat{K})+\boldsymbol{x} P^{k}(\widehat{K}), \\
& \widehat{W}=P^{k}(\widehat{K}), \\
& \widehat{M}=\left\{\widehat{\mathrm{m}} \in L^{2}(\partial \widehat{K}):\left.\quad \widehat{\mathrm{m}}\right|_{\widehat{e}} \in P^{k}(\widehat{e}) \quad \text { for all } \widehat{e} \in \partial \widehat{K}\right\} .
\end{aligned}
$$


Now, suppose we are given a symmetric positive definite $2 \times 2$ matrix function $\widehat{C}$, and a scalar non-negative function $\widehat{D}$ on $\widehat{K}$. For each $\widehat{\mathrm{m}}$ in $\widehat{M}$, we define the element $\left(\widehat{\boldsymbol{Q}}_{\widehat{\mathrm{m}}}, \widehat{U}_{\widehat{\mathrm{m}}}\right) \in \widehat{V} \times \widehat{W}$ by requiring that

$$
\begin{aligned}
& \int_{\widehat{K}} \widehat{C} \widehat{\boldsymbol{Q}}_{\widehat{\mathrm{m}}} \cdot \boldsymbol{V} d \widehat{\boldsymbol{x}}-\int_{\widehat{K}} \widehat{U}_{\widehat{\mathrm{m}}} \widehat{\nabla} \cdot \boldsymbol{V} d \widehat{\boldsymbol{x}}=-\sum_{\widehat{e} \in \partial \widehat{K}} \int_{\widehat{e}} \widehat{\mathrm{m}} \llbracket \boldsymbol{V} \rrbracket d \widehat{s}, \\
& \int_{\widehat{K}} W \widehat{\nabla} \cdot \widehat{\boldsymbol{Q}}_{\widehat{\mathrm{m}}} d \widehat{\boldsymbol{x}}+\int_{\widehat{K}} \widehat{D} \widehat{U}_{\widehat{\mathrm{m}}} W d \widehat{\boldsymbol{x}}=0,
\end{aligned}
$$

hold for all $(\boldsymbol{V}, W) \in \widehat{V} \times \widehat{W}$. Then, we have the following result.

Proposition 3.1. Let $K$ be any triangle and $e$ be one of its edges. Set

$$
\widehat{C}(\widehat{\boldsymbol{x}})=\left|\operatorname{det} D_{K}\right|^{-1} D_{K}^{t} c(\boldsymbol{x}) D_{K} \quad \text { and } \quad \widehat{D}(\widehat{\boldsymbol{x}})=\left|\operatorname{det} D_{K}\right| d(\boldsymbol{x}) .
$$

Then, the liftings $\boldsymbol{q}_{e, \ell}$ and $u_{e, \ell}$ on $K$ when mapped to $\widehat{K}$ satisfy

$$
\widehat{\boldsymbol{q}}_{e, \ell}=\widehat{\boldsymbol{Q}}_{\widehat{L_{e}, \ell}} \quad \text { and } \quad \widehat{u}_{e, \ell}=\widehat{U}_{\widehat{L_{e}, \ell}} .
$$

Moreover,

$$
\begin{aligned}
\mathbb{E}_{K ; e, \ell ; e^{\prime}, \ell^{\prime}} & =-\int_{\widehat{e}} \widehat{L_{e, \ell}} \widehat{\boldsymbol{Q}}_{\widehat{L_{e^{\prime}, \ell^{\prime}}}} \cdot \widehat{\boldsymbol{n}} d \widehat{s}, \\
\mathbb{H}_{g_{K ; e, \ell}} & =\int_{\widehat{e}} \widehat{g} \widehat{\boldsymbol{Q}}_{\widehat{L_{e, \ell}}} \cdot \widehat{\boldsymbol{n}} d \widehat{s} \\
\mathbb{H}_{f_{K ; e, \ell}} & =\int_{\widehat{K}} \widehat{U_{\widehat{L_{e}, \ell}}} \widehat{f}\left|\operatorname{det} D_{K}\right| d \widehat{\boldsymbol{x}} .
\end{aligned}
$$

This result can be easily proved by a straightforward change of variables and application of Lemma 2.1. Note that if the functions $\widehat{C}$ and $\widehat{D}$ are constant, there is no need to use quadrature rules to find the matrix entries.

\section{Comparison with the hybridized Brezzi-Douglas-Marini method}

Now we compare the multipliers given by the hybridized version of the RaviartThomas (RT) method and those given by the hybridized version of the corresponding Brezzi-Douglas-Marini (BDM) method.

4.1. Statement of the results. To state our comparison results, we first introduce the hybridized BDM method. The approximate solution given by this method, $\left(\boldsymbol{q}_{h}^{\mathrm{BDM}}, u_{h}^{\mathrm{BDM}}, \lambda_{h}^{\mathrm{BDM}}\right)$, is sought in the finite element space $V_{h}^{\mathrm{BDM}} \times W_{h}^{\mathrm{BDM}} \times M_{h}$ given by

$$
\begin{aligned}
V_{h}^{\mathrm{BDM}} & =\left\{\boldsymbol{v} \in L^{2}(\Omega) \times L^{2}(\Omega):\left.\quad \boldsymbol{v}\right|_{K} \in P^{k}(K) \times P^{k}(K), \quad \text { for all } K \in \mathcal{T}_{h}\right\}, \\
W_{h}^{\mathrm{BDM}} & =\left\{w \in L^{2}(\Omega):\left.\quad w\right|_{K} \in P^{k-1}(K), \quad \text { for all } K \in \mathcal{T}_{h}\right\},
\end{aligned}
$$


and is defined by requiring that, for all $(\boldsymbol{v}, u, \mu) \in V_{h}^{\mathrm{BDM}} \times W_{h}^{\mathrm{BDM}} \times M_{h}$,

$$
\begin{aligned}
& \int_{\Omega} c \boldsymbol{q}_{h}^{\mathrm{BDM}} \cdot \boldsymbol{v} d x-\sum_{K \in \mathcal{T}_{h}} \int_{K} u_{h}^{\mathrm{BDM}} \nabla \cdot \boldsymbol{v} d x+\sum_{e \in \mathcal{E}_{i, h}} \int_{e} \lambda_{h}^{\mathrm{BDM}} \llbracket \boldsymbol{v} \rrbracket d s=-\int_{\partial \Omega} g \llbracket \boldsymbol{v} \rrbracket d s, \\
& \sum_{K \in \mathcal{T}_{h}} \int_{K} w \nabla \cdot \boldsymbol{q}_{h}^{\mathrm{BDM}} d x+\int_{\Omega} d u_{h}^{\mathrm{BDM}} w d x=\int_{\Omega} f w d x, \\
& \sum_{e \in \mathcal{E}_{i, h}} \int_{e} \mu \llbracket \boldsymbol{q}_{h}^{\mathrm{BDM}} \rrbracket d s=0 .
\end{aligned}
$$

Note that the approximate solution of the BDM method satisfies exactly the same weak formulation than the approximate solution of the RT method; the only difference being the choice of the finite element spaces. As a consequence, the characterization theorem (Theorem 2.1) holds for the hybridized version of the BDM method. This is the key fact that allows us to compare the hybridized versions of the RT and the BDM methods.

In comparing RT and BDM methods, for the sake of readability, we shall superscript the notations previously introduced in connection with the Raviart-Thomas method by "RT". When superscripted by "BDM", such notations are to be understood as defined exactly as before except that the BDM spaces are substituted for the RT spaces. For example, $\left(\boldsymbol{q}_{h}, u_{h}\right)_{\mathrm{m}}^{\mathrm{BDM}} \equiv\left(\boldsymbol{q}_{h, \mathrm{~m}}^{\mathrm{BDM}}, u_{h, \mathrm{~m}}^{\mathrm{BDM}}\right) \in V_{h}^{\mathrm{BDM}} \times W_{h}^{\mathrm{BDM}}$ is defined by requiring that

$$
\begin{aligned}
& \int_{\Omega} c \boldsymbol{q}_{h, \mathrm{~m}}^{\mathrm{BDM}} \cdot \boldsymbol{v} d x-\sum_{K \in \mathcal{T}_{h}} \int_{K} u_{h, \mathrm{~m}}^{\mathrm{BDM}} \nabla \cdot \boldsymbol{v} d x=-\sum_{e \in \mathcal{E}_{h}} \int_{e} \mathrm{~m} \llbracket \boldsymbol{v} \rrbracket d s \\
& \sum_{K \in \mathcal{T}_{h}} \int_{K} w \nabla \cdot \boldsymbol{q}_{h, \mathrm{~m}}^{\mathrm{BDM}} d x+\int_{\Omega} d u_{h, \mathrm{~m}}^{\mathrm{BDM}} w d x=0
\end{aligned}
$$

hold for all $(\boldsymbol{v}, w) \in V_{h}^{\mathrm{BDM}} \times W_{h}^{\mathrm{BDM}}$.

To state our first comparison theorem, we need the following additional notation: Denote by $\mathcal{P}_{k}$ the $L^{2}$-orthogonal projection into the space of functions which are piecewise polynomials of degree $k$ on each triangle $K \in \mathcal{T}_{h}$. Let $\mathcal{R} f=\mathcal{P}_{k} f-\mathcal{P}_{k-1} f$ for all $f \in L^{2}(\Omega)$. Define the form

$$
b_{h, \mathcal{R} f}^{\mathrm{RT}}(\mu)=\int_{\Omega} \mathcal{R} f u_{h, \mu}^{\mathrm{RT}} d x, \quad \text { for all } \mu \in M_{h},
$$

and the function $\rho_{h} \equiv \rho_{h}(\mathcal{R} f) \in M_{h}$ by

$$
a_{h}^{\mathrm{RT}}\left(\rho_{h}, \mu\right)=b_{h, \mathcal{R} f}^{\mathrm{RT}}(\mu), \quad \text { for all } \mu \in M_{h} .
$$

Also set

$$
\begin{aligned}
\Psi(\mu, g, f) & =\left(\mathbf{0}, \mathcal{R} u_{h, \mu}^{\mathrm{RT}}\right)+\left(\mathbf{0}, \mathcal{R} u_{h, g}^{\mathrm{RT}}\right)+\left(\mathbf{0}, \mathcal{R} u_{h, \mathcal{P}_{k-1} f}^{\mathrm{RT}}\right), \quad \text { and } \\
\Upsilon(\mathcal{R} f) & =\left(\boldsymbol{q}_{h}, u_{h}\right)_{\rho_{h}}^{\mathrm{RT}}+\left(\boldsymbol{q}_{h}, u_{h}\right)_{\mathcal{R} f}^{\mathrm{RT}} .
\end{aligned}
$$

Now we can state the theorem.

Theorem 4.1 (Comparison of the RT and BDM methods for $d=0$ ). Assume that $d(\boldsymbol{x})=0$ almost everywhere in $\Omega$. 
(1) Suppose $f \in L^{2}(\Omega)$ is such that $\left(\mathcal{P}_{k}-\mathcal{P}_{k-1}\right) f=0$. Then, the Lagrange multiplier components of the Raviart-Thomas and Brezzi-Douglas-Marini solutions coincide:

$$
\lambda_{h}^{\mathrm{RT}}=\lambda_{h}^{\mathrm{BDM}} .
$$

(2) If $f \in L^{2}(\Omega)$ is arbitrary, then the following statements hold:

$$
\begin{array}{ll}
(\alpha) & a_{h}^{\mathrm{RT}}(\mathrm{m}, \mu)=a_{h}^{\mathrm{BDM}}(\mathrm{m}, \mu), \quad \text { for all } \mathrm{m}, \mu \in L^{2}\left(\mathcal{E}_{h}\right) . \\
(\beta) & b_{h}^{\mathrm{RT}}(\mu)=b_{h}^{\mathrm{BDM}}(\mu)+b_{h, \mathcal{R} f}^{\mathrm{RT}}(\mu), \quad \text { for all } \mu \in L^{2}\left(\mathcal{E}_{h}\right) . \\
(\gamma) & \lambda_{h}^{\mathrm{RT}}=\lambda_{h}^{\mathrm{BDM}}+\rho_{h}(\mathcal{R} f) . \\
(\delta) & \left(\boldsymbol{q}_{h}^{\mathrm{RT}}, u_{h}^{\mathrm{RT}}\right)=\left(\boldsymbol{q}_{h}^{\mathrm{BDM}}, u_{h}^{\mathrm{BDM}}\right)+\Psi\left(\lambda_{h}^{\mathrm{BDM}}, g, f\right)+\Upsilon(\mathcal{R} f) .
\end{array}
$$

Before proving the theorem, let us discuss the result and some of its consequences.

Remark 4.1. Statements $(\alpha)$ and $(\beta)$ can be easily rewritten in matrix form as follows:

$$
\begin{aligned}
& \left(\alpha^{\prime}\right) \quad \mathbb{E}^{\mathrm{RT}}=\mathbb{E}^{\mathrm{BDM}}, \\
& \left(\beta^{\prime}\right) \quad \mathbb{H}^{\mathrm{RT}}=\mathbb{H}^{\mathrm{BDM}}+\mathbb{H}_{\mathcal{R} f}^{\mathrm{RT}},
\end{aligned}
$$

where the matrix $\mathbb{E}^{\mathrm{N}}$ is the stiffness matrix associated to the bilinear form $a_{h}^{\mathrm{N}}(\cdot, \cdot)$, and the matrix $\mathbb{H}^{\mathrm{N}}$ is the right-hand side matrix associated to the linear form $b_{h}^{\mathrm{N}}(\cdot)$, for ${ }_{\mathrm{N}} \in\{\mathrm{RT}, \mathrm{BDM}\}$. The matrix $\mathbb{H}_{\mathcal{R} f}^{\mathrm{RT}}$ is, of course, the right-hand side matrix associated to the linear form $b^{\mathrm{RT}}(\cdot)_{h, \mathcal{R} f}$. This means that, when $d=0$, the stiffness matrices of the multipliers of both methods coincide. However, the right hand side matrices differ. But they differ by a matrix which vanish when $\mathcal{R} f=0$, hence the coincidence of the Lagrange multipliers whenever $\mathcal{R} f=0$.

Remark 4.2. The coincidence of Lagrange multipliers asserted by the theorem in the case $\mathcal{R} f=0$ (and $d=0$ ) appears to have gone unnoticed hitherto even numerically. This case occurs, e.g., when $f$ is a polynomial of degree $k-1$ on every element of the mesh, and in several applications of practical interest, e.g., incompressible flow in porous media (where $f=0$ usually). The condition $\mathcal{R} f=0$ is not only sufficient, but also necessary for such a coincidence: From the characterization theorem, it is clear that the only part of $f$ that determines $\lambda_{h}^{\mathrm{RT}}$ is $\mathcal{P}_{k} f$, while the only part of $f$ that determines $\lambda_{h}^{\mathrm{BDM}}$ is $\mathcal{P}_{k-1} f$. Therefore, setting $f$ to a polynomial of degree $k$ for which $0=\mathcal{P}_{k-1} f \neq \mathcal{P}_{k} f$, we can make $\lambda_{h}^{\mathrm{RT}} \neq \lambda_{h}^{\mathrm{BDM}}$.

Remark 4.3. Statement $(\delta)$ of the theorem shows how the solution components other than the multipliers are related. Obviously, $\Upsilon(\mathcal{R} f)$ depends linearly on $\mathcal{R} f$. Therefore, when $\mathcal{R} f=0$, the solution $\left(\boldsymbol{q}_{h}^{\mathrm{RT}}, u_{h}^{\mathrm{RT}}\right)$ differs from $\left(\boldsymbol{q}_{h}^{\mathrm{BDM}}, u_{h}^{\mathrm{BDM}}\right)$ only by $\Psi\left(\lambda_{h}^{\mathrm{BDM}}, g, f\right)$, a function that can be computed locally element by element. In particular, this means that it is possible to implement the less expensive BDM method and locally recover the RT solution $u_{h}^{\mathrm{RT}}$ which is one order higher in accuracy (under certain regularity assumptions). In this sense, $(\delta)$ can be thought of as yielding a postprocessing technique. Of course, one can then further postprocess the RT solution by the technique of [1] and gain one further order in accuracy.

Remark 4.4. It is well known that the Lagrange multiplier of both the RT and the BDM methods approximate the traces of the exact solution $u$ on mesh edges. Specifically, under certain regularity assumptions, [5, Lemma 4.1] asserts that when 
$\mathcal{T}_{h}$ is a quasiuniform mesh with mesh size $h$, as $h \rightarrow 0$,

$$
\left\|\lambda_{h}^{\mathrm{BDM}}-\mathcal{P}_{M_{h}} u\right\|_{\mathcal{E}_{h}}=O\left(h^{k+3 / 2-\delta_{k 1}}\right),
$$

where $\mathcal{P}_{M_{h}}$ denotes the $L^{2}$-orthogonal projection onto $M_{h},\|\cdot\|_{\mathcal{E}_{h}}$ denotes the $L^{2}\left(\mathcal{E}_{h}\right)$-norm, and $\delta_{k 1}$ is zero for all $k$ except for $k=1$ in which case it equals one. The analogous estimate for the RT method [1, Corollary 1.5] is

$$
\left\|\lambda_{h}^{\mathrm{RT}}-\mathcal{P}_{M_{h}} u\right\|_{\mathcal{E}_{h}}=O\left(h^{k+3 / 2}\right) .
$$

But obviously whenever $\lambda_{h}^{\mathrm{RT}}=\lambda_{h}^{\mathrm{BDM}}$ there can be no difference in the convergence rates. Therefore, when $k=1$ and $\mathcal{R} f=0$, by virtue of Theorem 4.1, we conclude that although (4.1) only provides for $O\left(h^{3 / 2}\right)$-convergence, in fact the convergence rate is at least $O\left(h^{5 / 2}\right)$.

When $d \neq 0$, the liftings $\boldsymbol{q}_{h, \mathrm{~m}}^{\mathrm{RT}}$ and $\boldsymbol{q}_{h, \mathrm{~m}}^{\mathrm{BDM}}$ are no longer divergence free on each element, in general. In fact, as we show later, there are multipliers $\mathrm{m}$ for which $\mathcal{R} u_{h, \mathrm{~m}}^{\mathrm{RT}} \neq 0$. This property implies that the statements of Theorem 4.1 do not hold in general. In particular, the following theorem provides a case wherein Statement $(\alpha)$ does not hold. Obviously, whenever Statement $(\alpha)$ fails to hold, one cannot expect coincidence of RT and BDM Lagrange multipliers.

Theorem 4.2 (Comparison of the RT and BDM methods for $d \geq 0$ ). Assume that $c(\boldsymbol{x})$ and $d(\boldsymbol{x})$ are constant on each element of the mesh. Then, whenever $d(\boldsymbol{x})$ is positive on at least one element, Statement $(\alpha)$ of Theorem 4.2 does not hold.

Now, we prove Theorems 4.1 and 4.2 .

4.2. Proof of Theorem 4.1. The proof proceeds by establishing a connection between the BDM and RT liftings and applying the characterization theorem. Two properties of the finite element spaces of the RT and BDM methods play a crucial role: The first is simply that the multipliers of both methods share the same space. The second is that the elements of $V_{h}^{\mathrm{RT}}$ whose divergence on each element is a polynomial of degree $k-1$ also belong to the space $V_{h}^{\mathrm{BDM}}$. Let us begin by proving the latter property.

Lemma 4.1. The following containment holds:

$$
\left\{\boldsymbol{q}_{h} \in V_{h}^{\mathrm{RT}}:\left.\nabla \cdot \boldsymbol{q}_{h}\right|_{K} \in P^{k-1}(K), \text { for all } K \in \mathcal{T}_{h}\right\} \subset V_{h}^{\mathrm{BDM}} .
$$

Proof. If $\boldsymbol{q}_{h} \in V_{h}^{\mathrm{RT}}$ then $\left.\boldsymbol{q}_{h}\right|_{K}=\boldsymbol{v}_{k}+\boldsymbol{x} \widetilde{p}_{k}$ for some $\boldsymbol{v}_{k} \in P^{k}(K) \times P^{k}(K)$ and some homogeneous polynomial $\widetilde{p}_{k}(\boldsymbol{x})$ of degree $k$ on $K$. Taking divergence, we find that

$$
\nabla \cdot\left(\left.\boldsymbol{q}_{h}\right|_{K}\right)=\nabla \cdot \boldsymbol{v}_{k}+(k+2) \widetilde{p}_{k} .
$$

Therefore, $\nabla \cdot\left(\left.\boldsymbol{q}_{h}\right|_{K}\right) \in P^{k-1}(K)$ implies that $\widetilde{p}_{k}=0$, and consequently $\left.\boldsymbol{q}_{h}\right|_{K} \in$ $P^{k}(K) \times P^{k}(K)$. Hence $\boldsymbol{q}_{h} \in V_{h}^{\mathrm{BDM}}$.

The next result uses the above lemma and the definition of the liftings to establish key relations between the liftings of the RT and BDM methods.

Lemma 4.2. Assume that $d=0$. Then, for all $\mathrm{m} \in L^{2}\left(\mathcal{E}_{h}\right)$ and $f \in L^{2}(\Omega)$,

$$
\begin{array}{lll}
\text { (i) } \quad \boldsymbol{q}_{h, \mathrm{~m}}^{\mathrm{RT}}=\boldsymbol{q}_{h, \mathrm{~m}}^{\mathrm{BDM}}, & u_{h, \mathrm{~m}}^{\mathrm{RT}}=u_{h, \mathrm{~m}}^{\mathrm{BDM}}+\mathcal{R} u_{h, \mathrm{~m}}^{\mathrm{RT}}, \\
\text { (ii) } & \boldsymbol{q}_{h, f}^{\mathrm{RT}}=\boldsymbol{q}_{h, f}^{\mathrm{BDM}}+\boldsymbol{q}_{h, \mathcal{R} f}^{\mathrm{RT}}, & u_{h, f}^{\mathrm{RT}}=u_{h, f}^{\mathrm{BDM}}+\mathcal{R} u_{h, \mathcal{P}_{k-1} f}^{\mathrm{RT}}+u_{h, \mathcal{R} f}^{\mathrm{RT}} .
\end{array}
$$


Proof. Let us begin by proving (i). From Equation (2.9), we have,

$\int_{\Omega} c\left(\boldsymbol{q}_{h, \mathrm{~m}}^{\mathrm{RT}}-\boldsymbol{q}_{h, \mathrm{~m}}^{\mathrm{BDM}}\right) \cdot \boldsymbol{v} d x-\sum_{K \in \mathcal{T}_{h}} \int_{K}\left(u_{h, \mathrm{~m}}^{\mathrm{RT}}-u_{h, \mathrm{~m}}^{\mathrm{BDM}}\right) \nabla \cdot \boldsymbol{v} d x=0, \quad$ for all $\boldsymbol{v} \in V_{h}^{\mathrm{BDM}}$,

and from the equation (2.10), $\left.\nabla \cdot \boldsymbol{q}_{h, \mathrm{~m}}^{\mathrm{RT}}\right|_{K}=0$, and $\left.\nabla \cdot \boldsymbol{q}_{h, \mathrm{~m}}^{\mathrm{BDM}}\right|_{K}=0$, for all $K \in \mathcal{T}_{h}$. Since by Lemma 4.1, $\boldsymbol{q}_{h, \mathrm{~m}}^{\mathrm{RT}} \in V_{h}^{\mathrm{BDM}}$, we can take $\boldsymbol{v}=\boldsymbol{q}_{h, \mathrm{~m}}^{\mathrm{RT}}-\boldsymbol{q}_{h, \mathrm{~m}}^{\mathrm{BDM}}$ in the first equation of this proof to get that $\boldsymbol{q}_{h, \mathrm{~m}}^{\mathrm{RT}}=\boldsymbol{q}_{h, \mathrm{~m}}^{\mathrm{BDM}}$. It immediately follows that

$$
-\sum_{K \in \mathcal{T}_{h}} \int_{K}\left(u_{h, \mathrm{~m}}^{\mathrm{RT}}-u_{h, \mathrm{~m}}^{\mathrm{BDM}}\right) \nabla \cdot \boldsymbol{v} d x=0, \quad \text { for all } \boldsymbol{v} \in V_{h}^{\mathrm{BDM}},
$$

which implies that $\mathcal{P}_{k-1} u_{h, \mathrm{~m}}^{\mathrm{RT}}=u_{h, \mathrm{~m}}^{\mathrm{BDM}}$. This proves $(i)$.

Now, let us prove $(i i)$. It suffices to show that for $p=\mathcal{P}_{k-1} f$,

$$
\boldsymbol{q}_{h, p}^{\mathrm{RT}}=\boldsymbol{q}_{h, p}^{\mathrm{BDM}}, \quad \text { and } \quad u_{h, p}^{\mathrm{RT}}=u_{h, p}^{\mathrm{BDM}}+\mathcal{R} u_{h, p}^{\mathrm{RT}} .
$$

Indeed, once we have (4.2), by linearity and the obvious equality

$$
\left(\boldsymbol{q}_{h}, u_{h}\right)_{\mathcal{P}_{k-1} f}^{\mathrm{BDM}}=\left(\boldsymbol{q}_{h}, u_{h}\right)_{f}^{\mathrm{BDM}},
$$

we get that

$$
\begin{aligned}
\left(\boldsymbol{q}_{h}, u_{h}\right)_{f}^{\mathrm{RT}} & =\left(\boldsymbol{q}_{h}, u_{h}\right)_{p}^{\mathrm{RT}}+\left(\boldsymbol{q}_{h}, u_{h}\right)_{\mathcal{R} f}^{\mathrm{RT}} \\
& =\left(\boldsymbol{q}_{h}, u_{h}\right)_{p}^{\mathrm{BDM}}+\left(\mathbf{0}, \mathcal{R} u_{h, p}^{\mathrm{RT}}\right)+\left(\boldsymbol{q}_{h}, u_{h}\right)_{\mathcal{R} f}^{\mathrm{RT}},
\end{aligned}
$$

and (ii) follows. To show (4.2), first observe that since $\left.p\right|_{K}=\left.\left(\mathcal{P}_{k-1} f\right)\right|_{K} \in$ $P^{k-1}(K)$, Equation (2.12), implies that

$$
\left.\nabla \cdot \boldsymbol{q}_{h, p}^{\mathrm{RT}}\right|_{K}=p, \quad \text { and }\left.\quad \nabla \cdot \boldsymbol{q}_{h, p}^{\mathrm{BDM}}\right|_{K}=p, \quad \text { for all } K \in \mathcal{T}_{h} .
$$

Therefore, using Lemma 4.1 again, $\boldsymbol{q}_{h, p}^{\mathrm{RT}} \in V_{h}^{\mathrm{BDM}}$. Now, (2.11) yields

$$
\int_{\Omega} c\left(\boldsymbol{q}_{h, p}^{\mathrm{RT}}-\boldsymbol{q}_{h, p}^{\mathrm{BDM}}\right) \cdot \boldsymbol{v} d x-\sum_{K \in \mathcal{T}_{h}} \int_{K}\left(u_{h, p}^{\mathrm{RT}}-u_{h, p}^{\mathrm{BDM}}\right) \nabla \cdot \boldsymbol{v} d x=0, \quad \text { for all } \boldsymbol{v} \in V_{h}^{\mathrm{BDM}} .
$$

Since we have shown that $\boldsymbol{q}_{h, p}^{\mathrm{RT}} \in V_{h}^{\mathrm{BDM}}$, we can choose $\boldsymbol{v}=\boldsymbol{q}_{h, p}^{\mathrm{RT}}-\boldsymbol{q}_{h, p}^{\mathrm{BDM}}$ above. Then the first equality of (4.2), namely $\boldsymbol{q}_{h, p}^{\mathrm{RT}}=\boldsymbol{q}_{h, p}^{\mathrm{BDM}}$, immediately follows. The second equality of (4.2) also follows, because by $(i)$, we have $\mathcal{P}_{k-1} u_{h, p}^{\mathrm{RT}}=u_{h, p}^{\mathrm{BDM}}$. This completes the proof.

We are now ready to prove Theorem 4.1.

Proof of Theorem 4.1. First of all, observe that the first conclusion of the theorem, namely $\lambda_{h}^{\mathrm{RT}}=\lambda_{h}^{\mathrm{BDM}}$ whenever $\mathcal{R} f=0$, follows from Statement $(\gamma)$, because $\rho_{h}(\mathcal{R} f)$ depends linearly on $\mathcal{R} f$. Therefore, we shall only prove Statements $(\alpha)-(\delta)$.

Applying the characterization theorem with $d=0$, we find that

$$
a_{h}^{\mathrm{N}}(\mathrm{m}, \mu)=\int_{\Omega} c \boldsymbol{q}_{h, \mathrm{~m}}^{\mathrm{N}} \cdot \boldsymbol{q}_{h, \mu}^{\mathrm{N}}, \quad \text { for } \mathrm{N} \in\{\mathrm{RT}, \mathrm{BDM}\},
$$

so the equality of $(\alpha)$ follows from the first equality of Lemma 4.2(i). Similarly, since

$$
b_{h}^{\mathrm{N}}(\mu)=\int_{\partial \Omega} g \llbracket \boldsymbol{q}_{h, \mu}^{\mathrm{N}} \rrbracket d s+\int_{\Omega} f u_{h, \mu}^{\mathrm{N}} d x, \quad \text { for } \mathrm{N} \in\{\mathrm{RT}, \mathrm{вDM}\},
$$

the second equality, namely $(\beta)$, also follows from Lemma $4.2(i)$. Now, Statement $(\gamma)$ obviously follows from $(\alpha),(\beta)$, and the definitions of the multipliers. 
To prove Statement $(\delta)$, we start again from the characterization theorem, apply Statement $(\gamma)$, and use the identities of Lemma 4.2 in succession:

$$
\begin{aligned}
\left(\boldsymbol{q}_{h}^{\mathrm{RT}}, u_{h}^{\mathrm{RT}}\right)= & \left(\boldsymbol{q}_{h}, u_{h}\right)_{\lambda_{h}^{\mathrm{RT}}}^{\mathrm{RT}}+\left(\boldsymbol{q}_{h}, u_{h}\right)_{g}^{\mathrm{RT}}+\left(\boldsymbol{q}_{h}, u_{h}\right)_{f}^{\mathrm{RT}} \\
= & \left(\boldsymbol{q}_{h}, u_{h}\right)_{\left(\lambda_{h}^{\mathrm{BDM}}+\rho_{h}\right)}^{\mathrm{RT}}+\left(\boldsymbol{q}_{h}, u_{h}\right)_{g}^{\mathrm{RT}}+\left(\boldsymbol{q}_{h}, u_{h}\right)_{f}^{\mathrm{RT}} \\
= & \left(\boldsymbol{q}_{h}, u_{h}\right)_{\lambda_{h}^{\mathrm{BDM}}}^{\mathrm{BDM}}+\left(\mathbf{0}, \mathcal{R} u_{h, \lambda_{h}^{\mathrm{RDM}}}^{\mathrm{RT}}+u_{h, \rho_{h}}^{\mathrm{RT}}\right)+\left(\boldsymbol{q}_{h, g}^{\mathrm{BDM}}, u_{h, g}^{\mathrm{BDM}}+\mathcal{R} u_{h, g}^{\mathrm{RT}}\right) \\
& \quad+\left(\boldsymbol{q}_{h, f}^{\mathrm{BDM}}+\boldsymbol{q}_{h, \mathcal{R} f}^{\mathrm{RT}}, u_{h, f}^{\mathrm{BDM}}+\mathcal{R} u_{h, \mathcal{P}_{k-1}}^{\mathrm{RT}}+u_{h, \mathcal{R} f}^{\mathrm{RT}}\right) \\
= & \left(\boldsymbol{q}_{h}, u_{h}\right)_{\lambda_{h}^{\mathrm{BDM}}}^{\mathrm{BDM}}+\left(\boldsymbol{q}_{h}, u_{h}\right)_{g}^{\mathrm{BDM}}+\left(\boldsymbol{q}_{h}, u_{h}\right)_{f}^{\mathrm{BDM}} \\
& \quad+\Psi\left(\lambda_{h}^{\mathrm{BDM}}, g, f\right)+\Upsilon(\mathcal{R} f) .
\end{aligned}
$$

The first three terms on the right hand side of the last equality sum to the BDM solution. This completes the proof of Theorem 4.1.

4.3. Proof of Theorem 4.2. To prove this result, we begin by studying the local spaces of the RT method, namely,

$$
\begin{aligned}
& V_{K}^{\mathrm{RT}}=P^{k}(K) \times P^{k}(K)+\boldsymbol{x} P^{k}(K), \\
& W_{K}^{\mathrm{RT}}=P^{k}(K), \\
& M_{K}^{\mathrm{RT}}=\left\{\mu \in L^{2}(\partial K):\left.\mu\right|_{e} \in P^{k}(e) \text { for each edge of } K\right\} .
\end{aligned}
$$

It turns out that, when $d>0$, the lifting operators induce a natural orthogonal decomposition of the local space $V_{K}^{\mathrm{RT}}$, namely,

$$
V_{K}^{\mathrm{RT}}=V_{K}^{0} \oplus V_{K}^{\perp},
$$

where

$$
\begin{aligned}
& V_{K}^{0}=\left\{\boldsymbol{v} \in V_{K}^{\mathrm{RT}}: \boldsymbol{v} \cdot \boldsymbol{n}_{e}=0 \text { on each edge } e \text { of } K\right\} \\
& V_{K}^{\perp}=\left\{\boldsymbol{v} \in V_{K}^{\mathrm{RT}}:((\boldsymbol{v}, \boldsymbol{q}))=0 \text { for all } \boldsymbol{q} \in V_{K}^{0}\right\} .
\end{aligned}
$$

and

$$
\left(\left(\boldsymbol{v}_{1}, \boldsymbol{v}_{2}\right)\right)=\int_{K} c \boldsymbol{v}_{1} \cdot \boldsymbol{v}_{2} d x+\int_{K} \frac{1}{d} \nabla \cdot \boldsymbol{v}_{1} \nabla \cdot \boldsymbol{v}_{2} d x .
$$

Indeed, the following result states that the local space $V_{K}^{\perp}$ is nothing but the image of the lifting operator $\mathrm{m} \mapsto \boldsymbol{q}_{h, \mathrm{~m}}^{\mathrm{RT}}$.

Lemma 4.3. Assume that $d(\boldsymbol{x})$ is a positive constant on an element $K \in \mathcal{T}_{h}$ and let $\left\{\mathrm{m}_{i}\right\}_{i=1}^{3(k+1)}$ be a basis of $M_{K}^{\mathrm{RT}}$. Then, $\left\{\boldsymbol{q}_{h, \mathrm{~m}_{i}}^{\mathrm{RT}}\right\}_{i=1}^{3(k+1)}$ is a basis of $V_{K}^{\perp}$.

Proof. By (2.10), we have for any $\mathrm{m} \in M_{K}^{\mathrm{RT}}$,

$$
u_{h, \mathrm{~m}}^{\mathrm{RT}}=-\frac{1}{d} \nabla \cdot \boldsymbol{q}_{h, \mathrm{~m}}^{\mathrm{RT}} .
$$

Substituting this expression for $u_{h, \mathrm{~m}}^{\mathrm{RT}}$ into (2.9), we see that (2.9) can be rewritten as follows:

$$
\left(\left(\boldsymbol{q}_{h, \mathrm{~m}}^{\mathrm{RT}}, \boldsymbol{v}\right)\right)=-\sum_{e \in \partial K} \int_{e} \mathrm{~m} \llbracket \boldsymbol{v} \rrbracket d s .
$$

As a consequence

$$
\left\{\boldsymbol{q}_{h, \mathrm{~m}_{i}}^{\mathrm{RT}}\right\}_{i=1}^{3(k+1)} \subset V_{K}^{\perp} .
$$


Since the dimension of $V_{K}^{\perp}$ is $3(k+1)$, it only remains to show that the elements of the set $\left\{\boldsymbol{q}_{h, \mathrm{~m}_{i}}^{\mathrm{RT}}\right\}_{i=1}^{3(k+1)}$ are linearly independent. So, assume that there are scalars $\alpha_{i}$ such that

$$
\sum_{i=1}^{3(k+1)} \alpha_{i} \boldsymbol{q}_{h, \mathrm{~m}_{i}}^{\mathrm{RT}}=0 .
$$

By the linearity of the lifting, this implies that $\boldsymbol{q}_{h, \mathrm{~m}}^{\mathrm{RT}}=0$, where

$$
\mathrm{m}=\sum_{i=1}^{3(k+1)} \alpha_{i} \mathrm{~m}_{i}
$$

But since, by (4.3),

$$
u_{h, \mathrm{~m}}^{\mathrm{RT}}=-\frac{1}{d} \nabla \cdot \boldsymbol{q}_{h, \mathrm{~m}}^{\mathrm{RT}}=0,
$$

we have that both the liftings $\boldsymbol{q}_{h, \mathrm{~m}}^{\mathrm{RT}}$ and $u_{h, \mathrm{~m}}^{\mathrm{RT}}$ are zero. Consequently, $\mathrm{m}=0$. Since $\left\{\mathrm{m}_{i}\right\}_{i=1}^{3(k+1)}$ is a basis for $M_{K}^{\mathrm{RT}}$, it follows that $\alpha_{i}=0$ for all $i=1, \ldots, 3(k+1)$. This completes the proof.

Now, we use the above result to show that the lifting $u_{h, \mathrm{~m}}^{\mathrm{RT}}$ is not always a polynomial of degree at most $k-1$ on all the elements of the triangulation.

Lemma 4.4. Assume that $c(\boldsymbol{x})$ is constant on an element $K \in \mathcal{T}_{h}$. Also assume that $d(\boldsymbol{x})$ is a positive constant on $K$. Then there is a function $\mathrm{m} \in M_{K}^{\mathrm{RT}}$ such that

$$
\mathcal{R} u_{h, \mathrm{~m}}^{\mathrm{RT}} \neq 0 \quad \text { on } K \text {. }
$$

Proof. Since, by (4.3),

$$
u_{h, \mathrm{~m}}^{\mathrm{RT}}=-\frac{1}{d} \nabla \cdot \boldsymbol{q}_{h, \mathrm{~m}}^{\mathrm{RT}},
$$

an application of Lemma 4.1 shows that $\mathcal{R} u_{h, \mathrm{~m}}^{\mathrm{RT}}=0$ if and only if

$$
\boldsymbol{q}_{h, \mathrm{~m}}^{\mathrm{RT}} \in P^{k}(K) \times P^{k}(K) \quad \text { for all } \mathrm{m} \in M_{K}^{\mathrm{RT}} .
$$

We claim that this is not possible for all $\mathrm{m} \in M_{K}^{\mathrm{RT}}$. Indeed, if this were the case, $V_{K}^{\perp} \subset P^{k}(K) \times P^{k}(K)$. This implies that the orthogonal complement of $P^{k}(K) \times P^{k}(K)$ in $V_{K}^{\mathrm{RT}}$ with respect to the innerproduct $((\cdot, \cdot))$, which we denote by $W_{K}$, satisfies

$$
W_{K} \subset V_{K}^{0}
$$

However, as we shall now see, this implies that $W_{K}=\{\mathbf{0}\}$, a contradiction.

In the orthogonality relation

$$
\int_{K} c \boldsymbol{\phi} \cdot \boldsymbol{v} d x+\frac{1}{d} \int_{K}(\nabla \cdot \phi)(\nabla \cdot \boldsymbol{v}) d x=0, \quad \text { for all } \boldsymbol{v} \in P^{k}(K) \times P^{k}(K),
$$

let us choose $\boldsymbol{v}=c^{-T} \nabla \eta$ for some $\eta \in P^{k+1}(K)$ (where $c^{-T}$ denotes the inverse of the transpose of $c$ ):

$$
\int_{K} \phi \cdot \nabla \eta d x+\frac{1}{d} \int_{K}(\nabla \cdot \phi)\left(\nabla \cdot c^{-T} \nabla \eta\right) d x=0 .
$$

By (4.5), and integration by parts,

$$
\int_{K} \boldsymbol{\phi} \cdot \nabla \eta d x+\int_{K} \eta \nabla \cdot \boldsymbol{\phi} d x=\int_{\partial K} \eta(\boldsymbol{\phi} \cdot \boldsymbol{n}) d s=0 .
$$


Subtracting (4.6) from (4.7) we have

$$
\int_{K}\left(\eta-\frac{1}{d} \nabla \cdot c^{-T} \nabla \eta\right)(\nabla \cdot \phi) d x=0, \quad \text { for all } \eta \in P^{k+1}(K) .
$$

Now we show that (4.8) implies that $\nabla \cdot \phi=0$. Choosing $\eta \in P^{1}(K)$ in (4.8), we conclude that $\nabla \cdot \phi$ is $L^{2}(K)$-orthogonal to $P^{1}(K)$. For $k \geq 2$, if $\nabla \cdot \phi$ is $L^{2}(K)$-orthogonal to $P^{k-1}(K)$, then choosing $\eta \in P^{k}(K)$ we find that $\nabla \cdot \phi$ is $L^{2}(K)$-orthogonal to $P^{k}(K)$ as well, because $\nabla \cdot\left(c^{-T} \nabla \eta\right) \in P^{k-2}(K)$. Thus, by induction, $\nabla \cdot \phi$ is zero.

It follows from $\nabla \cdot \phi=0$ that $\phi=0$ : Indeed, any $\phi \in W$ can be written as $\phi(\boldsymbol{x})=\boldsymbol{x} \widetilde{p}_{k}-\mathcal{Q}_{k}\left(\boldsymbol{x} \widetilde{p}_{k}\right)$, where $\mathcal{Q}_{k}$ is the orthogonal projection onto $P^{k}(K) \times P^{k}(K)$ in the $\left((\cdot, \cdot)\right.$ )-innerproduct, and $\widetilde{p}_{k}$ is a homogeneous polynomial of degree $k$. Therefore

$$
0=\nabla \cdot \phi=(k+2) \widetilde{p}_{k}-\nabla \cdot \mathcal{Q}_{k}\left(\boldsymbol{x} \widetilde{p}_{k}\right) .
$$

Since the latter term is in $P^{k-1}(K)$, we conclude that $\widetilde{p}_{k}=0$, so $\boldsymbol{\phi}=\mathbf{0}$. Thus, (4.4) does not hold, and the lemma is proved.

The next result establishes an equivalent criterion for Statement $(\alpha)$ of Theorem 4.1 in terms of the liftings.

Lemma 4.5. Assume that $d(\boldsymbol{x})$ is constant on every element of the mesh. Let $\mathrm{m} \in L^{2}(\partial K)$. Then

if and only if

$$
a_{h}^{\mathrm{RT}}(\mathrm{m}, \mathrm{m})=a_{h}^{\mathrm{BDM}}(\mathrm{m}, \mathrm{m}),
$$

(i) $\quad \boldsymbol{q}_{h, \mathrm{~m}}^{\mathrm{RT}}=\boldsymbol{q}_{h, \mathrm{~m}}^{\mathrm{BDM}}, \quad$ on $\Omega$, and

(ii) $u_{\mathrm{m}}^{\mathrm{RT}}=u_{\mathrm{m}}^{\mathrm{BDM}}, \quad$ on all elements $K \in \mathcal{T}_{h}$ where $d>0$.

Proof. Set $\mathbb{J}(\mathrm{m})=a_{h}^{\mathrm{RT}}(\mathrm{m}, \mathrm{m})-a_{h}^{\mathrm{BDM}}(\mathrm{m}, \mathrm{m})$. Since, by Theorem 2.1, we have, for $\mathrm{N} \in\{\mathrm{RT}, \mathrm{BDM}\}$,

$$
a_{h}^{\mathrm{N}}(\mathrm{m}, \mathrm{m})=\int_{\Omega} c \boldsymbol{q}_{h, \mathrm{~m}}^{\mathrm{N}} \cdot \boldsymbol{q}_{h, \mathrm{~m}}^{\mathrm{N}} d x+\int_{\Omega} d u_{h, \mathrm{~m}}^{\mathrm{N}} u_{h, \mathrm{~m}}^{\mathrm{N}} d x,
$$

a straightforward use of the identity $a^{2}-b^{2}=(a-b)^{2}+2 b(a-b)$ allows us to write $\mathbb{J}(m)=\Theta(m)+\mathbb{D}(m)$, where

$$
\Theta(\mathrm{m})=\int_{\Omega} c\left(\boldsymbol{q}_{h, \mathrm{~m}}^{\mathrm{RT}}-\boldsymbol{q}_{h, \mathrm{~m}}^{\mathrm{BDM}}\right) \cdot\left(\boldsymbol{q}_{h, \mathrm{~m}}^{\mathrm{RT}}-\boldsymbol{q}_{h, \mathrm{~m}}^{\mathrm{BDM}}\right) d x+\int_{\Omega} d\left(u_{\mathrm{m}}^{\mathrm{RT}}-u_{\mathrm{m}}^{\mathrm{BDM}}\right)^{2} d x .
$$

and

$$
\mathbb{D}(\mathrm{m})=2 \int_{\Omega} c \boldsymbol{q}_{h, \mathrm{~m}}^{\mathrm{BDM}} \cdot\left(\boldsymbol{q}_{h, \mathrm{~m}}^{\mathrm{RT}}-\boldsymbol{q}_{h, \mathrm{~m}}^{\mathrm{BDM}}\right) d x+2 \int_{\Omega} d u_{h, \mathrm{~m}}^{\mathrm{BDM}}\left(u_{h, \mathrm{~m}}^{\mathrm{RT}}-u_{h, \mathrm{~m}}^{\mathrm{BDM}}\right) d x .
$$

We will now show that $\mathbb{D}(m)=0$. Consider the first term in the definition of $\mathbb{D}(\mathrm{m})$. By $(2.9)$,

$$
\begin{aligned}
\int_{\Omega} c \boldsymbol{q}_{h, \mathrm{~m}}^{\mathrm{BDM}} \cdot\left(\boldsymbol{q}_{h, \mathrm{~m}}^{\mathrm{RT}}-\boldsymbol{q}_{h, \mathrm{~m}}^{\mathrm{BDM}}\right) d x & =\int_{\Omega} c \boldsymbol{q}_{h, \mathrm{~m}}^{\mathrm{RT}} \cdot \boldsymbol{q}_{h, \mathrm{~m}}^{\mathrm{BDM}} d x-\int_{\Omega} c \boldsymbol{q}_{h, \mathrm{~m}}^{\mathrm{BDM}} \cdot \boldsymbol{q}_{h, \mathrm{~m}}^{\mathrm{BDM}} d x \\
& =-\sum_{K \in \mathcal{T}_{h}} \int_{K}\left(u_{h, \mathrm{~m}}^{\mathrm{RT}}-u_{h, \mathrm{~m}}^{\mathrm{BDM}}\right) \nabla \cdot \boldsymbol{q}_{h, \mathrm{~m}}^{\mathrm{BDM}} d x .
\end{aligned}
$$


Hence, using also (2.10), we have

$$
\begin{aligned}
\int_{\Omega} c \boldsymbol{q}_{h, \mathrm{~m}}^{\mathrm{BDM}} \cdot\left(\boldsymbol{q}_{h, \mathrm{~m}}^{\mathrm{RT}}-\boldsymbol{q}_{h, \mathrm{~m}}^{\mathrm{BDM}}\right) d x & =-\sum_{K \in \mathcal{T}_{h}} \int_{K}\left(\mathcal{P}_{k-1} u_{h, \mathrm{~m}}^{\mathrm{RT}}-u_{h, \mathrm{~m}}^{\mathrm{BDM}}\right) \nabla \cdot \boldsymbol{q}_{h, \mathrm{~m}}^{\mathrm{BDM}} d x \\
& =-\int_{\Omega} d u_{h, \mathrm{~m}}^{\mathrm{BDM}}\left(\mathcal{P}_{k-1} u_{h, \mathrm{~m}}^{\mathrm{RT}}-u_{h, \mathrm{~m}}^{\mathrm{BDM}}\right) d x .
\end{aligned}
$$

Inserting this expression in the definition of $\mathbb{D}$, we get

$$
\mathbb{D}(\mathrm{m})=2 \int_{\Omega} d u_{h, \mathrm{~m}}^{\mathrm{BDM}}\left(u_{h, \mathrm{~m}}^{\mathrm{RT}}-\mathcal{P}_{k-1} u_{h, \mathrm{~m}}^{\mathrm{RT}}\right) d x=0,
$$

because $d$ is constant on each element of the mesh. In other words, $\mathbb{J}(\mathrm{m})=\Theta(\mathrm{m})$.

This implies that $\mathbb{J}(m)=0$ if and only if $\Theta(m)=0$. The lemma follows from the definition of $\Theta(m)$, and the fact that $c(\boldsymbol{x})$ is positive definite.

We are now ready to prove Theorem 4.2.

Proof of Theorem 4.2. Since there is at least one element $K \in \mathcal{T}_{h}$ wherein $d$ is a positive constant, Lemma 4.4 asserts the existence of at least one function $\mathrm{m} \in M_{K}^{\mathrm{RT}}$ for which $\mathcal{R} u_{h, \mathrm{~m}}^{\mathrm{RT}} \neq 0$ on $K$. This implies that $u_{h, \mathrm{~m}}^{\mathrm{RT}} \neq u_{h, \mathrm{~m}}^{\mathrm{BDM}}$ on $K$. Therefore, by Lemma 4.5 , for any $\mu \in M_{h}$ such that $\left.\mu\right|_{\partial K}=\mathrm{m}$, we have $a_{h}^{\mathrm{RT}}(\mu, \mu) \neq a_{h}^{\mathrm{BDM}}(\mu, \mu)$. Consequently, Statement $(\alpha)$ of Theorem 4.1 does not hold.

\section{Concluding Remarks}

The characterization theorem obtained in this paper for the hybridized RaviartThomas method on triangular meshes, also holds for various other methods. For example, it holds for the Raviart-Thomas method on simplicial meshes in any space dimension, as well as on rectangular and cubic meshes. It also holds for the hybridized versions of the mixed methods of Brezzi-Douglas-Marini $[5,6]$ on rectangles, the method of Brezzi-Douglas-Durán-Fortin [3] on tetrahedra and bricks, and the method of Brezzi-Douglas-Fortin-Marini [4] on triangles, rectangles, tetrahedra and bricks.

As a consequence, the matrix entries for the multipliers of all of the above mentioned methods can be computed as described in Section 3. Moreover, a result similar to the comparison theorem (Theorem 4.1) holds for Raviart-Thomas and Brezzi-Douglas-Marini methods on multidimensional simplices. However, when the elements are rectangles or bricks, the subspace of divergence free members of the Raviart-Thomas and Brezzi-Douglas-Marini spaces on an element are not identical. Therefore, in general, we cannot expect an analogue of Theorem 4.1 to hold.

In a forthcoming paper, we show how to use the characterization result to obtain error estimates for the multipliers without relying on error estimates on the other variables, as it is customarily done. We also show how to use the characterization theorem to devise preconditioners for the multiplier equation.

Acknowledgments. The authors would like to thank Wolfgang Dahmen whose stimulating visit to the I.M.A., University of Minnesota, in the Spring of 2001, prompted them to explore ways of characterizing stiffness matrices of mixed methods by using discontinuous test functions. This paper is the unexpected outcome of such exploration. 


\section{REFERENCES}

1. D.N. Arnold and F. Brezzi, Mixed and non-conforming finite element methods: implementation, post-processing and error estimates, Modél. Math. Anal.Numér. 19 (1985), 7-35.

2. D.N. Arnold, F. Brezzi, B. Cockburn, and D. Marini, Unified analysis of discontinuous Galerkin methods for elliptic problems, SIAM J. Numer. Anal. 39 (2001), 1749-1779.

3. F. Brezzi, J. Douglas, Jr., R.E. Durán, and M. Fortin, Mixed finite element methods for second order elliptic problems in three variables, Numer. Math. 51 (1987), 237-250.

4. F. Brezzi, J. Douglas, Jr., M. Fortin, and D. Marini, Efficient mixed finite element methods in two and three space variables, Modél. Math. Anal.Numér. 21 (1987), 581-604.

5. F. Brezzi, J. Douglas, Jr., and D. Marini, Two families of mixed finite elements for second order elliptic problems, Numer. Math. 47 (1985), 217-235.

6. $\_$, Variable degree mixed methods for second order elliptic problems, Mat. Aplic. e Comp. 4 (1985), 19-34.

7. F. Brezzi, G. Manzini, D. Marini, P. Pietra, and A. Russo, Discontinuous finite elements for diffusion problems, Atti Convegno in onore di F. Brioschi (Milano 1997), Istituto Lombardo, Accademia di Scienze e Lettere, 1999, pp. 197-217.

8. _ Discontinuous Galerkin approximations for elliptic problems, Numerical Methods for Partial Differential Equations 16 (2000), 365-378.

9. B. Fraejis de Veubeke, Displacement and equilibrium models in the finite element method, Stress Analysis (O. C. Zienkiewicz and G. Holister, eds.), Wiley, New York, 1965.

10. L. D. Marini, An inexpensive method for the evaluation of the solution of the lowest order Raviart-Thomas mixed method, SIAM J. Numer. Anal. 22 (1985), 493-496.

11. P.A. Raviart and J.M. Thomas, A mixed method for second order elliptic problems, Mathematical Aspects of the Finite Element Method (I. Galligani and E. Magenes, eds.), Lecture Notes in Math. 606, Springer-Verlag, New York, 1977.

School of Mathematics, University of Minnesota, 206 Church Street S.E., MinneapoLIS, MN 55455, USA.

E-mail address: cockburn@math.umn.edu

Department of Mathematics, University of Florida, Gainesville, FL 32611-8105.

E-mail address: jayg@math.ufl.edu 